Supplement of J. Sens. Sens. Syst., 7, 101-111, 2018

https://doi.org/10.5194/jsss-7-101-2018-supplement

(C) Author(s) 2018. This work is distributed under

the Creative Commons Attribution 4.0 License.

(c) (1)

Supplement of

\title{
Design and evaluation of split-ring resonators for aptamer-based biosen- sors
}

Tobias Reinecke et al.

Correspondence to: Tobias Reinecke (reinecke@geml.uni-hannover.de)

The copyright of individual parts of the supplement might differ from the CC BY 4.0 License. 


\section{Sublementary material}

\section{Single calculation steps}

A quantitative description of the dependency between the extension of the electrical length $\Delta l$ and the split capacity $C$ can be found by calculating the input impedance of a transmission line of length $l_{0}$ terminated with the split capacity and equalizing this impedance with the input impedance of an open line (stub) of length $\lambda_{\text {res2 }}$.

According to transmission line theory, the input impedance of a transmission line with a length $l_{0}$ terminated with an impedance $Z_{2}$ at the resonance frequency $f_{\text {res } 2}$ is

$$
Z_{1}=Z_{L} \frac{Z_{2}+j Z_{L} \tan \left(2 \pi \frac{l_{0}}{\lambda_{\text {res } 2}}\right)}{Z_{L}+j Z_{2} \tan \left(2 \pi \frac{l_{0}}{\lambda_{\text {res } 2}}\right)}
$$

Here, $Z_{L}$ is the wave impedance of the resonator structure and $Z_{2}$ is the impedance of the split capacity, i.e.

$$
Z_{2}=\frac{1}{j 2 \pi f_{\text {res } 2} C}
$$

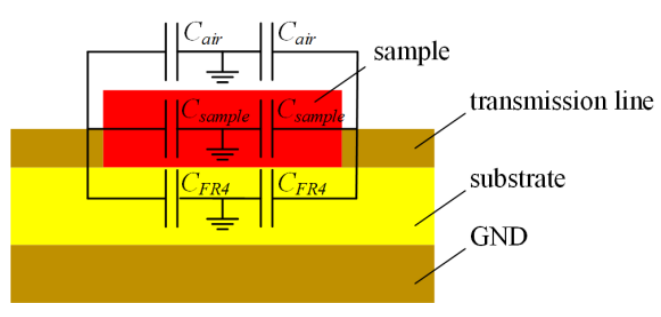

\section{Figure 1: Cross-section of the split capacity}

The electric potential in the center of the split is always $\varphi=0 \mathrm{~V}$, because the open ends of the resonator are always on opposite potential. Therefore, for modulating the split as an equivalent circuit, the cross-section depicted in Figure 1 shows a transmission line connected to ground via three parallel capacities: The sample dependent capacity $C_{\text {sample }}$ and two parasitic capacities above and below the sample capacity $C_{\text {air }}+C_{\text {substrate }}=C_{p}$.

Then, the split capacity $C$ can be written as

$$
C=C_{\text {sample }}+C_{p}=C_{\text {sample }, \varepsilon_{r}=1} \cdot \varepsilon_{r, \text { sample }}^{\prime}+C_{p} .
$$

Here, $C_{\text {sample,er }=1}$ is the sample capacity for an air filled split. Combining Eq. 1 and Eq. 2 yields 


$$
Z_{1}=Z_{L} \frac{\frac{1}{j 2 \pi f_{\text {res } 2} C}+j Z_{L} \tan \left(2 \pi \frac{l_{0}}{\lambda_{\text {res } 2}}\right)}{Z_{L}+j \frac{1}{j 2 \pi f_{\text {res } 2} C} \tan \left(2 \pi \frac{l_{0}}{\lambda_{\text {res } 2}}\right)}
$$

In the next step, $Z_{1}$ is equalized to the impedance of an open transmission line (see Eq. 1 with $Z_{2} \rightarrow \infty$ ) with length $l_{1}=\lambda_{\text {res } 2} / 4$ :

$$
Z_{1}=-j Z_{L} \cot \left(2 \pi \frac{l_{1}}{\lambda_{\text {res } 2}}\right)=-j Z_{L} \cot \left(2 \pi \frac{\lambda_{\text {res } 2}}{4 \lambda_{\text {res } 2}}\right)=-j Z_{L} \cot \left(\frac{\pi}{2}\right)=0 .
$$

From Eq. 4 with $Z_{1}=0$ follows

$$
\frac{1}{2 \pi f_{\text {res } 2} C}-Z_{L} \tan \left(2 \pi \frac{l_{0}}{\lambda_{\text {res } 2}}\right)=0 .
$$

Solving Eq. 6 for the resonance wave length $\lambda_{\text {res2 } 2 \text { yields }}$

$$
2 \pi l_{0} \frac{1}{\operatorname{arccot}\left(2 \pi f_{\text {res } 2} C Z_{L}\right)}=\lambda_{\text {res } 2} .
$$

With

$$
2 \pi f_{\text {res } 2}=\frac{2 \pi v}{\lambda_{\text {res } 2}}
$$

Eq. 8 becomes

$$
2 \pi l_{0} \frac{1}{\operatorname{arccot}\left(2 \pi \frac{v}{\lambda_{\text {res } 2}} C Z_{L}\right)}=\lambda_{\text {res } 2} .
$$

Here $v$ is the propagation velocity, which only depends on the speed of light $\mathrm{c}_{0}$ and the effective permittivity $\varepsilon_{\text {eff }}$, which in term is a superposition of the relative substrate permittivity $\left(\varepsilon_{\mathrm{r}, \mathrm{FR} 4}=4.1\right)$ and the relative permittivity of $\operatorname{air}\left(\varepsilon_{\mathrm{r}, \mathrm{Air}}=1\right)$ :

$$
v=\frac{c_{0}}{\sqrt{\varepsilon_{e f f}}} .
$$

To solve Eq. 9 for the desired resonance wave length, a taylor series approximation for $1 / \operatorname{arcot}(\mathrm{x})$ near 0 , which is aborted after the second element is performed, leading to the quadratic equation

$$
\lambda_{\text {res } 2}^{2}-4 l_{0} \lambda_{\text {res } 2}-16 l_{0} v C Z_{L}=0
$$

Solving Eq. 11 yields the desired dependency between resonance wave length $\lambda_{\text {res } 2}$ and the relative sample permittivty $\varepsilon_{r}$. 


$$
\lambda_{\text {res } 2}=2 l_{0}+\sqrt{4 l_{0}^{2}+16 l_{0} v C Z_{L}}=2 l_{0}+\sqrt{4 l_{0}^{2}+16 l_{0} v Z_{L}\left(C_{p}+C_{\text {sample }, \varepsilon_{r}=1} \cdot \varepsilon_{r, \text { sample }}^{\prime}\right)} .
$$

Note that when $\mathrm{C}=0$, Eq. 12 becomes

$$
\frac{\lambda_{r e s 2}}{4}=\frac{\lambda_{r e s 1}}{4}=l_{0} .
$$

This result supports the theory, because, as was already shown in Error! Reference source not found., for this hypothetical case, the half wave length of the resonance frequency must be equal to the circumference of the resonator.

\section{Changing the length of the finger structure}

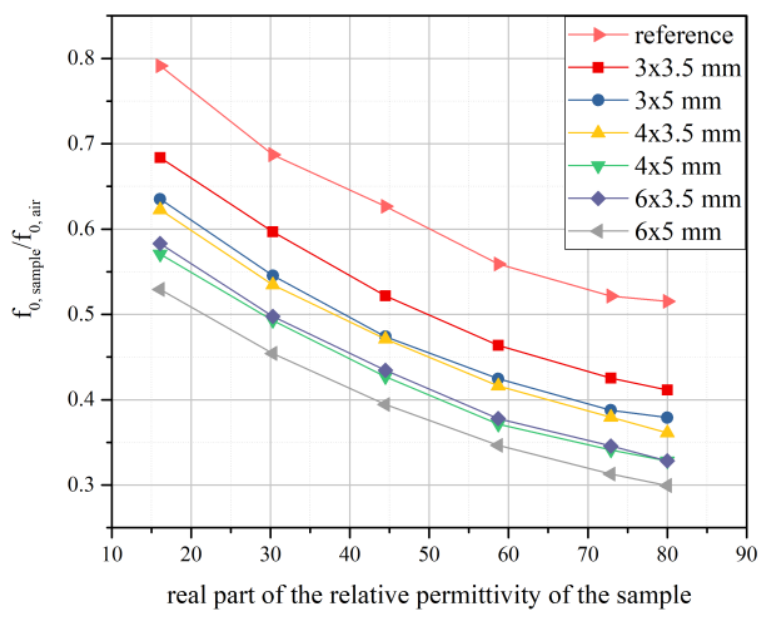

Figure S1: Measuring an increasing sample permittivity with different split capacities: Increasing either number of fingers or length of fingers increases the sensitivity, as the area of the split capacity increases. 